\title{
System Requirements for Optical HAP-Satellite Links
}

\author{
N. Perlot*, E. Duca**, J. Horwath*, D. Giggenbach * and E. Leitgeb ${ }^{* * *}$ \\ ${ }^{*}$ Institute of Communications and Navigation, DLR, Weßling, Germany \\ ** Department of Electronic Engineering, University of Rome "Tor Vergata", Italy \\ ${ }^{* * *}$ Institute of Broadband Communications, Graz University of Technology, Graz, Austria \\ nicolas.perlot@dlr.de, elisa.duca@uniroma2.it, joachim.horwath@dlr.de, \\ dirk.giggenbach@dlr.de, erich.leitgeb@tugraz.at
}

\begin{abstract}
Between a high-altitude platform (HAP) in the stratosphere and a satellite, high-data-rate optical links are conceivable but the challenges are still not clearly identified. We thus review the main requirements to properly establish such links. The design of a HAP-LEO link is in several aspects different from that of a HAP-GEO link. Among others, we consider the possible limitations caused by atmospheric propagation, the Sun and the environment of the HAP terminal. Signal transmission appears markedly more difficult when the optical beam must propagate near the HAP horizon or below.
\end{abstract}

\section{INTRODUCTION}

The high-altitude platform (HAP) is likely to be a key element in the development of free-space optics (FSO) technologies. FSO generally suffers from important drawbacks in the atmospheric environment. A HAP is located above the clouds where the air density is low; it is quasi-geostationary and may come down to the ground for maintenance. The clear sight of a HAP toward space is opportune for optical links with satellites.

Various communication scenarios can be considered. Large amount of data are generated on many LEO satellites and must be brought down to the Earth surface. The use of HAP as Earth-observation data relay has been investigated recently by several authors [1]-[4]. Data can also be generated by sensors onboard a HAP or may result from the reception by the HAP of several RF signals from the ground. In this context, an interesting scenario is the optical data transfer from one HAP to another over a relay GEO-satellite. The geo-stationarity of all terminals provides advantages: quasi-unlimited communication time, simplified tracking, constant received power and frequency. The possibility of implementing such a long link $(80000 \mathrm{~km})$ without any electrical conversion on the GEO satellite is investigated in [5]. Although two HAPs can be separated by hundreds of kilometres and still have a permanent line of sight, inter-HAP links are not directly addressed in this paper.

The feasibility of optical HAP links has been claimed many times. However the actual complexity of the implementation of reliable systems is not well known. This paper aims at identifying critical points specific to optical HAP-satellite links. We first review the basic characteristics of optical space communications. In Sect. III, the geometry of HAP links is detailed, as well as the importance of low-elevation links. Sect. IV deals with atmospheric-propagation effects. Sect. V examines the conditions under which the Sun can hinder a communication. The next sections are dedicated to requirements on spatial acquisition and on HAP terminals. The paper concludes with Sect. IX.

\section{BASic OpticAl SPACE System}

\section{A. Link Parameters}

For long-distance links, the optical beam should approach the ideal $\mathrm{TEM}_{00}$ Gaussian beam. The geometrical parameters of a link are represented in Fig. 1. "Tx" and "Rx" denote transmitter and receiver. $\theta_{d i v}$ is the $1 / \mathrm{e}^{2}$ half beam divergence, $L$ the propagation distance, and $D$ the receiver diameter. With $P_{T x}$ and $P_{R x}$ respectively the transmitted and received optical powers, the basic link equation is

$$
P_{R x}=P_{T x} \mho_{T x} \Omega_{R x} .
$$

$\mho_{T x}$ and $\Omega_{R x}$ are respectively the directivities of the transmitter and receiver with respect to isotropic antennas. For a transmitted Gaussian beam we have

$$
\mho_{T x}=\frac{8}{\theta_{d i v}^{2}}
$$

Note that the gain of a Gaussian beam is a factor 2 higher than that of a flat-top beam with half divergence $\theta_{d i v}$. Conversely, $\Omega_{R x}$ is given by the effective angular extent of the receiver:

$$
\Omega_{R x}=\left(\frac{D}{4 L}\right)^{2} .
$$

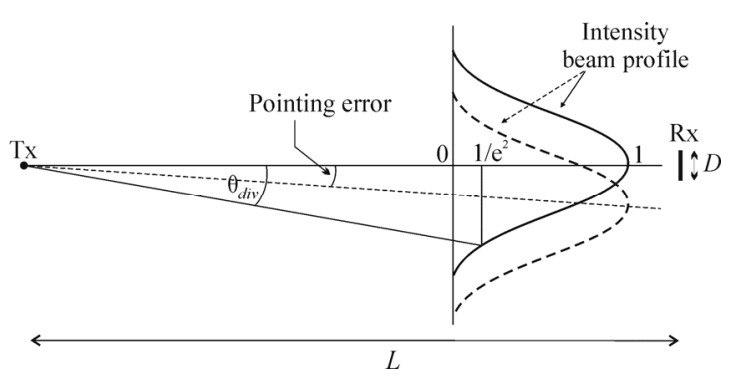

Fig. 1 Illustration of the link parameters. 
For both (2) and (3), the small-angle approximation has been used. The free-space loss that is explicitly defined in some other link budgets [6] is here contained in Eq. (3). To (1) must be added the possible losses and amplifications. As illustrated in Fig. 1, a pointing error leads to a misplacement of the beam footprint at the receiver. We note $\sigma_{\text {pointing }}$ the standard deviation of the pointing error on one axis and assume the one-axis pointing error is normally distributed. Calculating the loss due to pointing errors as a $10^{-2}$-probability fade, the pointing loss $L_{\text {pointing }}$ can be evaluated according to [7]

$$
L_{\text {pointing }}=10^{-2 \beta^{2}}
$$

where $\beta=2 \sigma_{\text {pointing }} / \theta_{d i v}$. Thus, for a given pointing error $\sigma_{\text {pointing }}$, the pointing loss $L_{\text {pointing }}$ decreases as one increases the beam divergence. On the other hand, increasing the beam divergence reduces $\boldsymbol{\mho}_{T x}$. The sum of $\boldsymbol{\mho}_{T x}$ (Eq. (2)) and $L_{\text {pointing }}$ (Eq. (4)) lets an optimum appear at $\theta_{d i v} \approx$ $5 \sigma_{\text {pointing }}$, or equivalently at $\beta \approx 0.4$ which gives a pointing loss of $L_{\text {pointing }}=-3.2 \mathrm{~dB}$.

\section{B. Current Trends}

Table 1 is a simplified power budget of a LEO-GEO link where $1 \mathrm{Gbit} / \mathrm{s}$ is achieved with $1 \mathrm{~W}$ transmitted power. Fig. 2 shows for various link distances the data rate achieved by the LCT terminals manufactured by Tesat Spacecom and operating at $1064 \mathrm{~nm}$ [8]. Oerlikon Space provides similar specifications for their terminals [9]. Data rates are limited essentially by (i) the available transmit power of communication lasers, (ii) the pointing errors and (iii) the telescope size. In space systems, pointing errors are determined by sensor resolutions and terminal vibrations. The telescope size is usually less than $300 \mathrm{~mm}$ to fulfil technical as well as financial requirements, especially when several terminals are to be placed on one satellite. As a result, the current divergence angle $\theta_{d i v}$ is

Table 1 Typical power budget of a 1-Gbit/s LEO-GEO link.

\begin{tabular}{|l|r|l|}
\hline Tx-Power (Average) & 1 & $\mathrm{~W}$ \\
\hline$\theta_{d i v}$ & 6.0 & $\mu \mathrm{rad}$ \\
\hline$L$ & 40000 & $\mathrm{~km}$ \\
\hline$D$ & 200 & $\mathrm{~mm}$ \\
\hline Tx-Power & 30.0 & $\mathrm{dBm}$ \\
\hline$\sigma_{T x}$ & 113.5 & $\mathrm{dBi}$ \\
\hline$\Omega_{R x}$ & -178.1 & $\mathrm{dBi}$ \\
\hline$L_{p o i n t i n g}$ & -3.2 & $\mathrm{~dB}$ \\
\hline Rx-Optics Loss & -3.0 & $\mathrm{~dB}$ \\
\hline Rx-Power & -40.8 & $\mathrm{dBm}$ \\
\hline $\begin{array}{l}\text { Required Rx-Power for } \\
1 \text { Gbit/s at BER }=1 \mathrm{E}-9\end{array}$ & -43.7 & $\mathrm{dBm}$ \\
\hline Link Margin & 2.9 & $\mathrm{~dB}$ \\
\hline
\end{tabular}

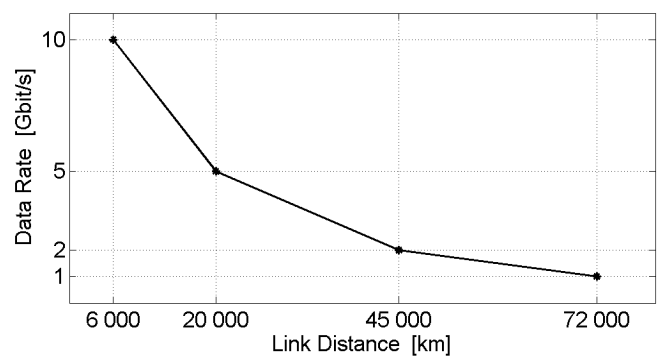

Fig. 2 Achievable data rate as a function of distance for the LCT terminals [LAN07]. These specifications are given for a BER of 1E-9. typically between 1 and $10 \mu \mathrm{rad}$. Note that to transmit e.g. a beam of wavelength $1550 \mathrm{~nm}$ with a divergence $\theta_{d i v}=1$ $\mu \mathrm{rad}$, a telescope of about $1-\mathrm{m}$ diameter would be required. Achieving reliable systems with sub- $\mu$ rad divergence is therefore challenging although conceivable.

\section{GEOMETRY OF HAP LINKS}

Because clouds block near-infrared beams, we consider only links above the clouds. The altitude of the cloud ceiling varies with latitudes. In temperate latitudes it is reasonable to set the cloud ceiling at $13-\mathrm{km}$ altitude. For simplicity, we will assume a spherical Earth and a constant maximum cloud altitude of $13 \mathrm{~km}$. Fig. 3 depicts the geometry of a link between a HAP and a satellite. A spherical satellite orbit is assumed. The angle $\alpha$ is the elevation of the link as seen from the HAP. The Earth angle $\phi$ is a function of satellite altitude $H_{\text {satellite, HAP }}$ altitude $H_{H A P}$ and elevation angle $\alpha$, i.e. we can write $\phi=$ $f\left(H_{\text {satellite }}, H_{H A P}, \alpha\right)$. For given altitudes $H_{\text {satellite }}$ and $H_{H A P}$, there exists a minimum elevation $\alpha_{\min }$ for which the link is tangent to the cloud ceiling. Corresponding to $\alpha_{\min }$, the maximum Earth angle $\phi_{\max }$ is given by $\phi_{\max }=f\left(H_{\text {satellite }}\right.$, $\left.H_{H A P}, \alpha_{\min }\right)$. As long as $H_{H A P}$ remains within typical altitudes (we consider here $13 \mathrm{~km}<H_{H A P}<25 \mathrm{~km}$ ), it has no big influence on $\phi$. However, $H_{H A P}$ has a significant influence on $\phi_{\max }$. To assess the importance of low elevation angles, we consider the relative coverage $c$ defined by the solid angle of radius $\phi$ normalized by that of radius $\phi_{\max }$ :

$$
c \equiv \frac{1-\cos (\phi)}{1-\cos \left(\phi_{\max }\right)} .
$$

For $H_{\text {satellite }}=600 \mathrm{~km}$ and $36000 \mathrm{~km}$, Fig. 4 and Fig. 5 respectively show the values taken by $c$ as a function of HAP altitude and link elevation. Clearly, for a higher satellite, low elevations are less important in terms of angular coverage.

Assuming a LEO satellite is present with an equal probability on each point of its spherical orbit surface delimited by $\phi_{\max }$, the probability that the HAP sees the satellite above a given elevation can be related to the coverage $c$. Looking at Fig. 4 with this assumption in mind, one can state that e.g. if a HAP at 20-km altitude can establish a link with a LEO at 600-km altitude only at

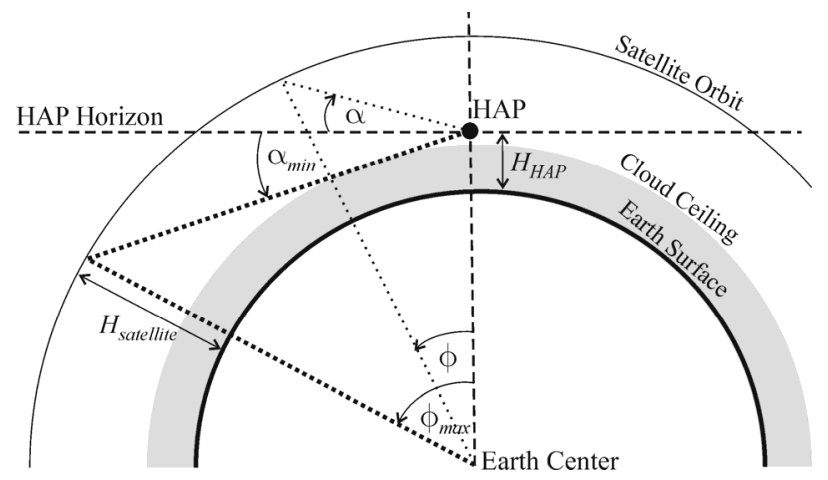

Fig. 3 Geometry of HAP-satellite links. 


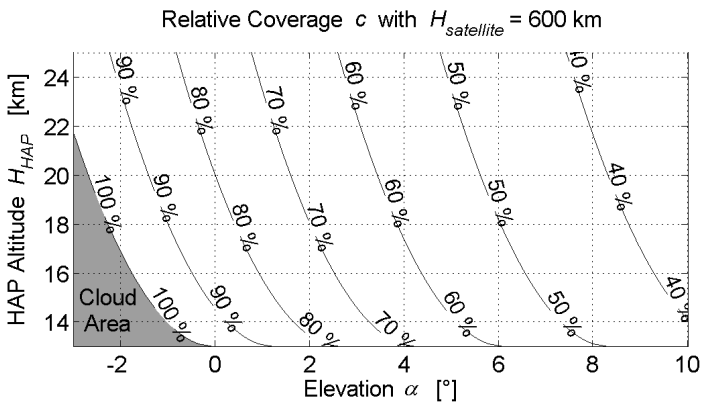

Fig. 4 Isolines of the relative coverage $c$ for $H_{\text {satellite }}=600 \mathrm{~km}$.

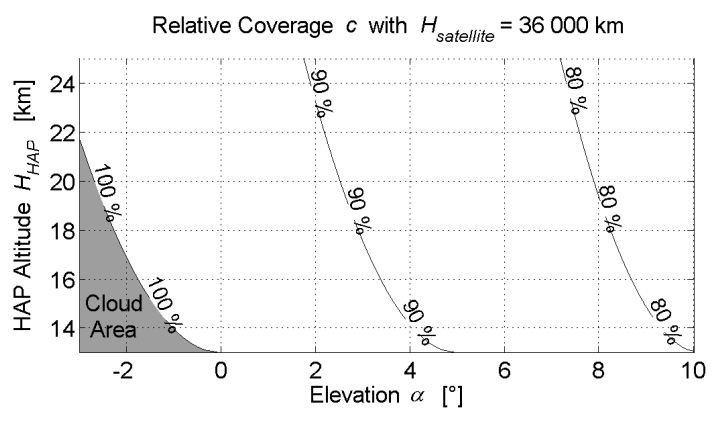

Fig. 5 Same as Fig. 4 but for $H_{\text {satellite }}=36000 \mathrm{~km}$.

elevations greater than $2^{\circ}$, about $32 \%$ of the potential link time (as defined by the cloud limit) is lost. Note that the assumption of equal probability over the orbital sphere does not always hold: a HAP located at a pole and communicating with polar-orbit satellites will see the satellites most often at zenith.

In the case of a HAP-GEO link, since both terminals are stationary, a different interpretation is required. In that case, one can relate variations in the elevation angle $\alpha$ to variations of the HAP position on the Earth with respect to the satellite position. Looking at Fig. 5, one can state that e.g. if a HAP at 20-km altitude can establish a link with a $G E O$ only at elevations greater than $2^{\circ}$, the area on Earth where the HAP can be placed is reduced by $9 \%$ (with respect to the cloud limit). The maximum latitude of the HAP is then $79.3^{\circ}$ whereas the maximum latitude set by the cloud limit was $84.0^{\circ}$.

\section{AtMOSPHERIC PROPAGATION}

\section{A. Optical Turbulence}

As the Gaussian beam depicted in Fig. 1 propagates through the atmosphere, optical turbulence distorts its wavefront and spatially redistributes its energy. The Hufnagel-Valley model is a widely-used vertical profile for the turbulence strength parameter $C_{n}^{2}$. We consider the so-called $\mathrm{HV}_{5 / 7}$ model (see e.g., [10] Sect. 12.2.1) which, for stratospheric heights, can be expressed as:

$$
C_{n}^{2}(h) \approx 3.6 \times 10^{-3}\left(10^{-5} h\right)^{10} \mathrm{e}^{-h / 1000} \quad h>7 \mathrm{~km} .(6)
$$

$h$ is the altitude in meter. $\mathrm{HV}_{5 / 7}$ corresponds to a moderately turbulent atmosphere. It is an averaged model and should be used only when turbulence is to be integrated over a path of at least several kilometers. For the sake of simplicity, we additionally assume isotropic turbulence. This is an approximation as measurements in the stratosphere reveal stronger vertical turbulence [11].
We consider here the coherence diameter $r_{0}$ which determines the Strehl ratio and affects the uplink as well as the downlink:

(i) For the uplink, when $r_{0}$ is on the same order of magnitude as the outgoing-beam radius $W_{0}$, significant beam wander takes place [10]. When $r_{0}$ is significantly smaller than $W_{0}$, a beam-spread effect takes place with a corresponding loss of approximately $r_{0}^{2} /\left(8 W_{0}^{2}\right)$.

(ii) For the downlink, angle-of-arrival fluctuations increase as $r_{0}$ decreases. When $r_{0}$ is significantly smaller than the receiving aperture diameter $D$, a great loss occurs in receivers designed to detect a single mode [14].

One may argue through the reciprocity principle [12] that the wander of the outgoing beam can be compensated by tracking the angle of the incoming beam. However, three facts weaken the validity of the reciprocity principle. First, the reciprocity principle is valid when turbulence is near the telescope. In the case of a negative-elevation link, most turbulence can be several hundreds of kilometres away from the HAP. Second, the point-ahead angle separating the incoming and outgoing beams (necessary to track fast-moving satellites) may be larger than the isoplanatic angle [13]. Finally, the sending aperture may not correspond to the receiving aperture. A small coherence diameter $r_{0}$ can therefore introduce pointing errors.

The coherence diameter is given by integrating the $C_{n}{ }^{2}$ parameter over the path (see e.g. [10] Sect. 12.4.1):

$$
r_{0}=2.1 \times\left[1.45 k^{2} \int_{0}^{L} C_{n}^{2}(h(z)) d z\right]^{-3 / 5}
$$

where $k$ is related to the wavelength $\lambda$ by $k=2 \pi / \lambda$. For $\lambda$ $=1 \mu \mathrm{m}$, Fig. 6 shows $r_{0}$ with respect to $H_{H A P}$ and $\alpha$. Considering $r_{0}=0.25 \mathrm{~m}$ with an aperture diameter of $D=$ $0.05 \mathrm{~m}$, the single-axis standard deviation of the angle of arrival would be approximately $1 \mu \mathrm{rad}$. In this case, turbulence may induce significant pointing errors.

Similar to wavefront distortions, scintillation (i.e., power fluctuations) is expected to be significant essentially at negative elevation angles.

\section{B. Attenuation}

Attenuation in the stratosphere is generally negligible at $1064 \mathrm{~nm}$, and even more at $1550 \mathrm{~nm}$. What can significantly enhance atmospheric extinction is the

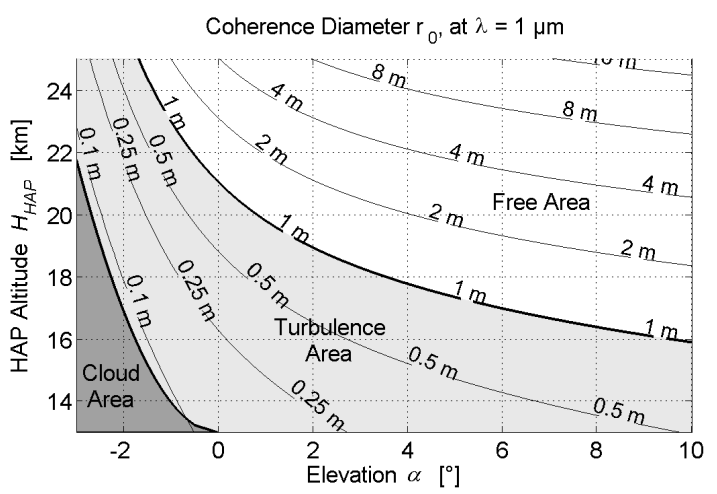

Fig. 6 Isolines of the coherence diameter $r_{0}$ calculated for the wavelength $\lambda=1 \mu \mathrm{m}$ and with the model $\mathrm{HV}_{5 / 7}$. 


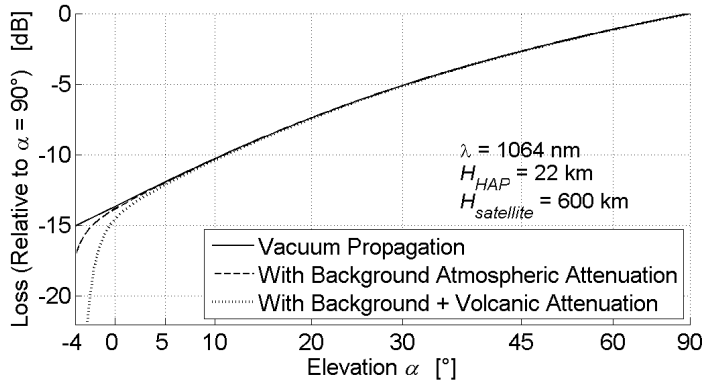

Fig. 7 Propagation loss as a function of elevation and normalized over the Zenith link $\left(\alpha=90^{\circ}\right)$ for $\lambda=1064 \mathrm{~nm}, H_{H A P}=22 \mathrm{~km}$ and $H_{\text {satellite }}=600$ km. [15]

presence of aerosols from volcanic activities. Fig. 7 gives losses that can be expected for $\lambda=1064 \mathrm{~nm}$ during a LEO overflight [15]. From $\alpha=90^{\circ}$ down to $0^{\circ}$, the loss in the received power is mostly due to the increase of the link distance. At an elevation of $-2^{\circ}$, one sees that the attenuation of the background atmosphere is less than 1 $\mathrm{dB}$ but cannot be neglected when volcanic particles are present on the path. Thus, counting with volcanic particles, the received optical power may reach a dynamic of $20 \mathrm{~dB}$ for a LEO overflight.

\section{SUN IMPACT}

The Sun is a strong background noise source that is likely to hinder a communication if its rays directly impinge on the detectors. The angular size of the Sun is about $2 \theta_{\text {sun }}=10 \mathrm{mrad}$. The Sun extent relative to a hemisphere is $\pi \theta_{\text {sun }}^{2} / 2 \pi \approx 10^{-5}$. Additionally, the receiver's field of view is generally smaller than the angular Sun size. Thus, the presence of the Sun in the field of view of the HAP terminal constitutes a rare event. If the Sun happens to be behind the satellite, the time during which the Sun remains in the HAP's field of view depends on the satellite orbit. This time is typically 2 minutes for a GEO satellite, whereas for a LEO satellite it is a few seconds. Regarding the satellite terminal, it may have the Sun in its field of view only at low link elevations, which essentially corresponds to sunrises and sunsets for the HAP.

Also, to prevent the Sun from heating the HAP terminal and deteriorating internal components, the terminal surface exposed to the Sun should be covered with reflecting material (like for space terminals).

\section{Spatial ACQUisition}

To acquire the direction to the counter-terminal with an accuracy on the order of a microradian, a specific acquisition procedure is necessary prior to any link. Each terminal knows, with a given accuracy, its position and orientation as well as the position of the counter-terminal. The accuracy of this knowledge defines the uncertainty cone containing the direction to the counter-terminal. The acquisition procedure depends on the size of the uncertainty cone, which in turn depends on the type of satellite (e.g. LEO or GEO) and on the type of HAP (aerostatic or aerodynamic).

Thanks to a GPS, the HAP terminal can determine its position with high accuracy. However, due to stratospheric winds the measured orientation (yaw, pitch, roll) of the HAP terminal will generally not be as precise as that of the satellite terminal. Also, to improve acquisition at low elevation, atmospheric refraction should be integrated in the terminal propagators as it deflects the beam from the straight path.

One can reasonably consider for both terminals an uncertainty-cone diameter of $1 \mathrm{mrad}$ dominated by attitude uncertainties. The emission of a strong beacon laser of 1-mrad divergence by one terminal would be the fastest acquisition method; however power on a HAP or satellite is limited and such a beacon may not be available. A different and longer acquisition procedure would consist in emitting a beacon of smaller divergence and scanning the uncertainty cone with this beacon. A long acquisition time is more problematic for HAP-LEO links than for HAP-GEO links since LEO overflights last typically only 10 minutes.

If the uncertainty cones happen to be asymmetrical, the beacon should clearly be sent by the terminal that knows best where to point at.

\section{TERMINAL ACCOMMODATION ON THE HAP}

In order to maximize the duration of HAP-LEO links (i.e., to have a link over large ranges of elevation and azimuth angles), the optical terminal should be the highest device on the HAP so that the line of sight is kept clear. E.g., if we consider an ellipsoidal balloon of length $120 \mathrm{~m}$ and width $50 \mathrm{~m}$, the terminal should be placed at least 20 $\mathrm{cm}$ above the balloon's top in order to have a panoramic sight down to $-3^{\circ}$ elevation. If the HAP is aerodynamic and flies with large roll angles, maintaining such a panoramic sight is more difficult. Performing several HAP-LEO links simultaneously complicate the problem as static terminals may obstruct one another. To reduce obstructions, a solution is to use redundant terminals on the HAP.

\section{HAP ENVIRONMENT}

The external temperature of a HAP terminal is $-50^{\circ} \mathrm{C}$ or less. Because the telescope temperature will probably be much higher, it is excluded that frost collects on mirrors or lenses. However it is important that the housing material and the devices in operation create a favourable temperature equilibrium within the terminal. An example of optical communication terminal tested in the stratosphere can be found in [16].

At $20-\mathrm{km}$ altitude, a great part of cosmic rays and radiations is still absorbed by the higher atmosphere. In this regard, qualification of components (e.g., fiber amplifiers) should be less strict for HAPs than for space systems.

\section{CONCLUSION}

Exploring the requirements of optical links between a HAP and a satellite, we found that most issues concern low elevations. Low elevations are particularly important for HAP-LEO links. To maximize the communication time with a LEO satellite, Ref. [4] proposes links down to the cloud ceiling (i.e., $\alpha=-2.7^{\circ}$ with $H_{H A P}=20 \mathrm{~km}$ ). To make such links reliable, some challenges must be surmounted. As the optical beam sinks toward the HAP horizon and below, one expects longer propagation distance, stronger atmospheric attenuation, stronger wavefront distortions and scintillation, larger Doppler shift, possible obstructions of the beam due to HAP geometry, and possible interference of the Sun on the 
satellite terminal. The system complexity is thus expected to grow along with the angular coverage of the HAP terminal.

\section{ACKNOWLEDGEMENT}

This work was supported by SatNEx II.

\section{REFERENCES}

[1] Cianca, E.; Prasad, R.; De Sanctis, M.; De Luise, A.; Antonini, M.; Teotino, D.; Ruggieri, M., "Integrated satellite-HAP systems", Communications Magazine, IEEE, Vol. 43, Issue 12, pp. 33-39 (2005)

[2] Antonini, M. Betti, S. Carrozzo, V. Duca, E. Ruggieri, M. "Feasibility analysis of a HAP-LEO optical link for data relay purposes ", Aerospace Conference, IEEE (2006)

[3] M. Knapek, J. Horwath, F. Moll, B. Epple, N. Courville, H. Bischl, D. Giggenbach, "Optical High-Capacity Satellite Downlinks via High-Altitude Platform Relays", Proc. SPIE 6709 67090E (2007)

[4] D. Giggenbach, J. Horwath, B. Epple, "Optical satellite downlinks to optical ground stations and high-altitude platforms," IST Mobile and Wireless Communication Summit, Budapest, July 2007.

[5] Betti, S; Carrozzo, V; Duca, E, "Over-Stratospheric-Altitude Optical Free Space Links: System Performance Evaluation Transparent Optical Networks", in ICTON, International Conference on Transparent Optical Networks, July 2007.

[6] S. Lambert, W. Casey: Laser communications in Space, Artech House Inc., Norwood, 1995

[7] K. Kiasaleh, "On the probability density function of signal intensity in free-space optical communications systems impaired by pointing jitter and turbulence," Opt. Eng., vol. 33, no. 11, pp. 3748-3757, 1994
[8] R. Lange, B. Smutny, "Homodyne BPSK-based optical intersatellite communication links", Proc. SPIE 6457, 645703 (2007)

[9] Guy Baister, Thomas Dreischer, Michael Tüchler, Klaus Kudielka, and Edgar Fischer, "OPTEL terminal for deep space telemetry links", Proc. SPIE 6457, 645706 (2007)

[10] L. Andrews, R. Phillips, Laser Beam Propagation through Random Media, $2^{\text {nd }}$ Edition, SPIE Press, Bellingham, WA, (2005).

[11] C. Robert, J. -M. Conan, V. Michau, J. -B. Renard, C. Robert, and F. Dalaudier, "Retrieving parameters of the anisotropic refractive index fluctuations spectrum in the stratosphere from balloon-borne observations of stellar scintillation," J. Opt. Soc. Am. A 25, 379393 (2008)

[12] D. L. Fried and H. T. Yura, "Telescope-performance reciprocity for propagation in a turbulent medium," J. Opt. Soc. Am. 62, 600602 (1972).

[13] D. L. Fried, "Anisoplanatism in adaptive optics," J. Opt. Soc. Am. 72, 52- (1982)

[14] N. Perlot, "Turbulence-induced fading probability in coherent optical communication through the atmosphere," Appl. Opt. 46, 7218-7226 (2007)

[15] B. Mayer, S. Shabdanov, D. Giggenbach, "Electronic Database of atmospheric absorption coefficients", DLR-internal report by DLR-IPA and DLR-IKN-DN-OCG, DLR-Oberpfaffenhofen, December 2002, based on the atmospheric constituents profiles according to "G.P. Anderson, et al: AFGL Atmospheric Constituent Profiles (0-120km), AFGL-TR-86-0110, Hanscom Air Force Base, MA 01736, 1986".

[16] J. Horwath, N. Perlot, M. Knapek, and F. Moll, "Experimental Verification of Optical Backhaul Links for High-Altitude Platform Networks: Atmospheric Turbulence and Downlink Availability", International Journal of Satellite Communications and Networking, Special Issue (2007) 